\title{
A comprehensive proteomic analysis of elaioplasts from citrus fruits reveals insights into elaioplast biogenesis and function
}

\author{
Man Zhu ${ }^{1,2}$, Jiajia Lin ${ }^{1,2}$, Junli Ye ${ }^{1,2}$, Rui Wang ${ }^{3}$, Chao Yang ${ }^{3}$, Jinli Gong ${ }^{1,2}$, Yun Liu ${ }^{1,2}$, Chongling Deng ${ }^{4}$, Ping Liu 4 , \\ Chuanwu Chen ${ }^{4}$, Yunjiang Cheng ${ }^{1,2}$, Xiuxin Deng $\mathbb{B}^{1,2}$ and Yunliu Zeng ${ }^{1,2}$
}

\begin{abstract}
Elaioplasts of citrus peel are colorless plastids which accumulate significant amounts of terpenes. However, other functions of elaioplasts have not been fully characterized to date. Here, a LC-MS/MS shotgun technology was applied to identify the proteins from elaioplasts that were highly purified from young fruit peel of kumquat. A total of 655 putative plastid proteins were identified from elaioplasts according to sequence homology in silico and manual curation. Based on functional classification via Mapman, $50 \%$ of the identified proteins fall into six categories, including protein metabolism, transport, and lipid metabolism. Of note, elaioplasts contained ATP synthase and ADP, ATP carrier proteins at high abundance, indicating important roles for ATP generation and transport in elaioplast biogenesis. Additionally, a comparison of proteins between citrus chromoplast and elaioplast proteomes suggest a high level of functional conservation. However, some distinctive protein profiles were also observed in both types of plastids notably for isoprene biosynthesis in elaioplasts, and carotenoid metabolism in chromoplasts. In conclusion, this comprehensive proteomic study provides new insights into the major metabolic pathways and unique characteristics of elaioplasts and chromoplasts in citrus fruit.
\end{abstract}

\section{Introduction}

Plants contain several kinds of plastids, such as chloroplasts in photosynthetic tissues (e.g., leaves and green fruit), chromoplasts in pigment-containing fruits, as well as colorless leucoplasts in starch-storing or lipid-storing seeds ${ }^{1}$. These plastids are reported as the sites for several essential processes, including carbon fixation, nitrogen assimilation, and the biosynthesis of isoprenoids, starches, fatty acids (FA), and amino acids. Although plastids are a family of organelles possessing the same plastid genome (encoding approximately 100 proteins), the majority (>95\%) of the 2000-3500 different proteins are imported

\footnotetext{
Correspondence: Yunliu Zeng (zengyl@mail.hzau.edu.cn)

${ }^{1}$ Key Laboratory of Horticultural Plant Biology (Ministry of Education), College of Horticulture and Forestry Science, Huazhong Agricultural University, Wuhan 430070, China

${ }^{2}$ Institute of Citrus Science, Huazhong Agricultural University, Wuhan 430070, China

Full list of author information is available at the end of the article
}

from the surrounding cytoplasm rather than being synthesized in $s i t u^{2,3}$. It is the imported proteins that define the developmental fate of plastids, leading to the different morphologies and special functions within specific plastids $^{4}$. Recently, large-scale proteomics analysis has become an efficient approach for the investigating subcellular organelles at the protein level ${ }^{2}$. Special attention has been given to chloroplasts as well as chromoplasts in several species, including tomato, citrus, pepper, papaya, watermelon, carrot, and orange curd cauliflower ${ }^{5-11}$. In contrast, relatively few proteomics studies have focused on other types of plastid.

Leucoplasts are colorless plastids that include amyloplasts, elaioplasts, and proteoplasts that store starches, lipids, or proteins, respectively. Amyloplasts have been well studied and have been shown to be enriched in many storage tissues, including seeds, tubers/root vegetables, and fruits. In contrast, elaioplasts are much less 
studied in plants. Besides their role in storage, elaioplasts were also reported to possess crucial biosynthetic functions, for example in the biosynthesis of long-chain FAs in developing castor seeds ${ }^{12}$ and terpenes in the outer peel of citrus ${ }^{13,14}$. Plastid isolation is a prerequisite not only for assessing proteome composition, but also for understanding metabolic processes, enzyme localization, metabolite transporters, and biogenesis. Although protocols for the isolation of elaioplasts are available for some plant tissues, including petals of snapdragon flowers (Antirrhinum majus) ${ }^{15}$, developing seeds of mustard (Brassica campestris) ${ }^{16,17}$, and maize (Zea mays) ${ }^{18}$, as well as the outer peel of citrus (Citrofortunella mitis $)^{14}$, the proteome profile of elaioplasts in these tissues is still unknown.

Citrus are one of the most important horticultural crops around the world, and have a unique anatomical structure, which consists of the pericarp (peel) and an edible endocarp (flesh). The endocarp accumulates large amounts of carotenoids within chromoplasts, which not only bring the red, yellow, and orange colors of fruit flesh, but also provide several important groups of nutrients. The pigmented region of pericarp contains active secretory cells that are characterized by numerous elaioplasts. Elaioplasts have been demonstrated to be involved in the biosynthesis of terpenes in essential oil, which are then exported into the secretory pocket, greatly affecting the aroma and taste of the fruit ${ }^{14,19}$. As the major component of essential oil, terpenes have been employed for manufacturing of perfumes and flavorings, and they are also used as antiseptic preservative agents to improve the safety and shelf-life of fruit during storage ${ }^{20,21}$. Although significant progress has been made in understanding chromoplast differentiation in the peel of citrus fruits ${ }^{22,23}$, little information is available about the regulatory mechanisms underlying elaioplast biogenesis.

In the present study, a comprehensive proteomic analysis using nLC-MS/MS-Q Exactive technology was used to identify proteins in elaioplasts isolated from the peel of kumquat by density gradient centrifugation. A total of 655 plastid protein candidates were detected that were predicted to participate in the general metabolism or unique metabolic pathways in elaioplasts, including flavor synthesis and fruit maturation. Overall, the present study provides an important insight into elaioplast biogenesis and formation of essential flavors.

\section{Materials and methods}

\section{Plant materials}

The outer peel of $\sim 150$ earlier-stage green fruits $(0.5-0.8 \mathrm{~cm}$ in diameter) of 'Rong An' kumquat (Fortunella margarita Swingle) was used for elaioplast isolation. Fresh mature pulp of 'Hong Anliu' orange ( $C$. sinensis) was used for chromoplast isolation. Both citrus cultivars were collected from Guangxi Institute of Citrus Research, located at Guilin city, Guangxi, China.

\section{Elaioplast and chromoplast purification}

Elaioplasts were purified according to the procedure described by Michel et al. ${ }^{14}$ with minor modifications. Approximately $10 \mathrm{~g}$ of tissue was cut into pieces at $4{ }^{\circ} \mathrm{C}$ and then gently homogenized in pre-cooled SPBTK buffer (50 mM Tricine- $\mathrm{NaOH}$ pH 7.8, $5 \mathrm{mM} \mathrm{KCl}, 0.3 \mathrm{M}$ sucrose, $0.5 \%(\mathrm{w} / \mathrm{v})$ soluble polyvinylpyrrolidone 360 , and $0.1 \%(\mathrm{w} /$ v) bovine serum albumin). The homogenized sample was passed through two layers of Micracloth (Calbiochem). The pooled filtrate was centrifuged for $8 \mathrm{~min}$ at $5000 \times g$. The pellet was carefully re-suspended in SPBTK buffer at $\mathrm{pH} 7$ and then centrifuged for $90 \mathrm{~s}$ at $800 \times g$. The resulting supernatant was centrifuged at $5000 \times g$ for 10 min. The suspension containing most of the plastids was gently layered onto the top of a discontinuous gradient of $0.75,0.92,1.20,1.50 \mathrm{M}$ sucrose in $50 \mathrm{mM}$ Tricine- $\mathrm{NaOH}$ $(\mathrm{pH}$ 6.5) and then centrifuged at $100,000 \times g$ for $90 \mathrm{~min}$ in a Hitachi P40ST rotor. Four zones, designated as bands 1 , 2, 3, 4 from the top to the bottom of the gradient, were clearly separated by sucrose gradient centrifugation. Each band was carefully recovered and examined under an optical microscope at $\times 100$ magnification (Cover-018; Olympus, Tokyo). The purity of each fraction was tested by western blot analysis (described below). The elaioplast layer (band 1) was recovered. Chromoplast isolation from sweet orange was performed as described previously ${ }^{10}$. The final pellets containing elaioplast or chromoplast fractions were collected and flash-frozen in liquid nitrogen and kept at $-80^{\circ} \mathrm{C}$. The plastid samples were prepared in two biological replicates.

\section{Western blot analysis}

Polyclonal antibodies were diluted as appropriate against plastid Rubisco large subunit (RubcL; $53 \mathrm{kDa}$, 1:3000), cytosolic UDP-glucose pyrophosphorylase (UDPase; $51.6 \mathrm{kDa}, 1: 2000$ ), vacuolar ATPase (V-ATPase; 26-31 kDa, 1:2000), and mitochondrial voltage-dependent anion-selective channel protein 1 (VDAC1; $29 \mathrm{kDa}$, 1:2000), as well as two photosynthesis-related antibodies (light-harvesting complex (Lhca1 and Lhca4), 1:5000) from Agrisera. A total of $30 \mu \mathrm{g}$ of proteins were used for immunoblot analysis as previously described ${ }^{10}$. At least two independent replicate experiments were performed for each immunoblot with similar result.

\section{Protein extraction and digestion in solution}

Protein extraction was performed as previously described $^{9}$. The filter-assisted sample preparation method was adapted for protein digestion. Briefly, $200 \mu \mathrm{g}$ of proteins were mixed with $100 \mu \mathrm{l}$ of $100 \mathrm{mM}$ dithiothreitol (DTT), $150 \mathrm{mM}$ Tris- $\mathrm{HCl} \mathrm{pH} \mathrm{8,4 \% (w/v)} \mathrm{SDS,} \mathrm{incubated} \mathrm{at} 100^{\circ}$ 


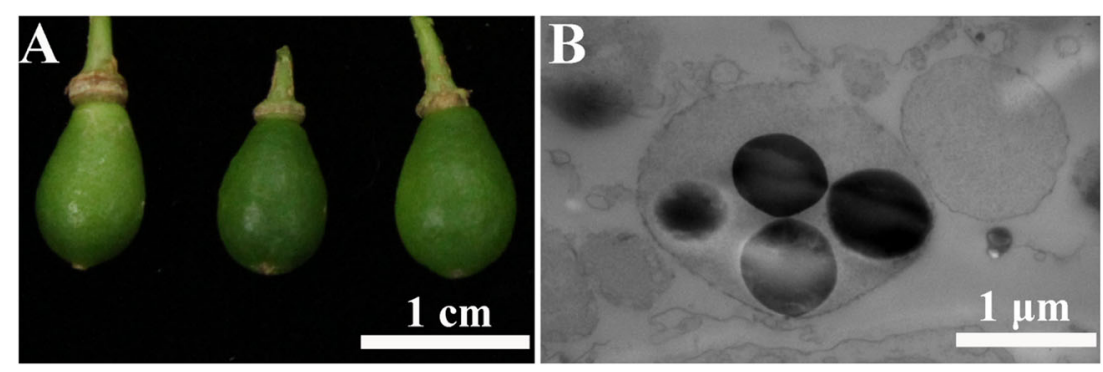

Fig. 1 Fruits of kumquat and elaioplasts in the outer peel. a A representative photograph of kumquat fruits used for elaioplasts isolation. b Transmission electron microscopic (TEM) picture showing the ultrastructural features of elaioplasts in the outer peel of kumquat

$\mathrm{C}$ for $10 \mathrm{~min}$, and then cooled to $25^{\circ} \mathrm{C}$. Next, UA buffer (150 mM Tris- $\mathrm{HCl}$, pH 8.0, $8 \mathrm{M}$ Urea) was used to remove detergent, DTT, and other low-molecular-mass substances by repeated ultrafiltrate $(10 \mathrm{kDa}$; Microcon units), and then centrifuged for $30 \mathrm{~min}$ at $14,000 \times g$. The samples were washed using $100 \mu \mathrm{l}$ of UA buffer followed by centrifugation for $30 \mathrm{~min}$ at $14,000 \times g$, and then incubated under darkness for $30 \mathrm{~min}$ by adding $100 \mu \mathrm{l}$ of UAI buffer (8 M Urea, $150 \mathrm{mM}$ Tris-HCl, pH 8.0, $50 \mathrm{mM}$ iodoacetamide). Further washes (three times) with $100 \mu \mathrm{l}$ of UA buffer followed by two washes using $100 \mu \mathrm{l}$ of 50 $\mathrm{mM} \mathrm{NH} \mathrm{NHCO}_{3}$ (pH 8.5) were performed. Finally, the resulting proteins were digested with $4 \mu \mathrm{g}$ of trypsin (Promega) overnight $(16-18 \mathrm{~h})$ at $37^{\circ} \mathrm{C}$. The concentration of peptide mix was estimated by UV absorbance at $280 \mathrm{~nm}$ as described previously ${ }^{9}$.

\section{Q Exactive-based LC-MS/MS analysis}

nLC-MS/MS analysis was performed on an Easy-nLC system equipped with a Thermo Scientific Q Exactive mass spectrometer. $5 \mu \mathrm{g}$ of peptide mixture was injected onto an Acclaim PepMap100 trap column (nanoViper C18, 100 $\mu \mathrm{m} \times 2 \mathrm{~cm}$, Thermo Scientific) fitted with a C18-reversed phase analytical column (Easy Column, $75 \mu \mathrm{m} \times 10 \mathrm{~cm}, 3$ $\mu \mathrm{m}$ resin, Thermo Scientific) in mobile phase A $(0.1 \%$ formic acid in water). Peptides were eluted with a linear 120 -min gradient of mobile phase B $(0.1 \%$ formic acid in $84 \%$ acetonitrile) at a flow rate of $0.3 \mu \mathrm{l} / \mathrm{min}$. The separated MS data were obtained as described in ref. ${ }^{9}$

MS/MS spectra were searched with the MaxQuant software package (version 1.1.0.36) against non-redundant protein databases of Citrus sinensis (http://www. pytozome.com or http://citrus.hzau.edu.cn), and the complete chloroplast genome sequence of $C$. sinensis (accession no. NC_008334). The search parameters were performed as described in ref. ${ }^{9}$ Only the proteins identified by two or more peptides in two bio-replicates were retained for further analysis.

\section{Functional categorization}

Detected proteins were categorized based on Mapman functional bins (https://mapman.gabipd.org/mapman). Additional curation was performed according to classifications in PPDB (http://ppdb.tc.cornell.edu). The proteins identified in citrus plastids were queried against three comprehensive plastid proteome databanks (AT_CHLORO, http://www.grenoble.prabi.fr/at_chloro/; PPDB (Plastid Proteome Database, http://ppdb.tc.cornell. $\mathrm{edu} /$ ); Plprot (Plastid Protein Database, http://www. plprot.ethz.ch/); downloaded in October 2016); and two plastidial protein subset databanks (Uniprot, http://www. uniprot.org/; SUBA, http://suba.plantenergy.uwa.edu.au/; downloaded in October 2016). There were 2816 plastid proteins in plprot, 1265 AT-CHLORO, 2292 in PPDB, 2977 in SUBA and 2038 in Uniprot.

Subcellular localization analysis was carried out using three software programs: Predotar (https:// urgi.versailles.inra.fr/predotar/), Target P (http://www. cbs.dtu.dk/services/TargetP/), and WoLF PSORT (http://www.genscript.com/wolf-psort.html). Proteins were tentatively assigned as plastidial (i) if predicted to be plastid targeted by at least one of the three programs, and/or (ii) if homologous to plastid proteins annotated in at least two plastidial databanks. Additionally, sequence information was manually curated if they were available in the literature. The putative plastid proteins of elaioplast and chromoplast were listed in Supplementary Table S1.

\section{Microscopy observation and analysis}

Isolated plastids were carefully layered onto microscope slides and visualized using a DP70 camera coupled to an Olympus BX61 microscope. Plastid samples for transmission electron microscopy (TEM) analysis were prepared and examined as described previously ${ }^{9}$. The plastid diameter was calculated by (minor axis + major axis) $/ 2$ with ImageJ (http://rsbweb.nih.gov/ij/). 


\section{Results and discussion}

Isolation of highly purified elaioplasts from kumquat peel with density gradient centrifugations

Kumquat, a major citrus cultivar in southern China, bears the smallest fruits in the Citrus genus. Ripened fruits can be eaten as a whole (Fig. 1a), and elaioplasts with several plastoglobules were frequently found in the outer peel (Fig. 1b).

In this study, we initially isolated elaioplasts from the outer peel of kumquat. The recovered pellet containing elaioplasts was separated into four fractions on a discontinuous gradient comprising 0.75, 0.92, 1.20, $1.50 \mathrm{M}$ sucrose (Fig. 2a). The elaioplasts pooled from band 1 were characterized by abundant colorless plastids with several sphere substructures clustering together, and their sizes ranged from 1 to $4 \mu \mathrm{m}$ as measured under light microscopy and TEM (Fig. 2b, c). Analysis of the other bands indicated they were contaminated by either cell debris with few elaioplasts, or chloroplasts (Supplementary Figure S1).

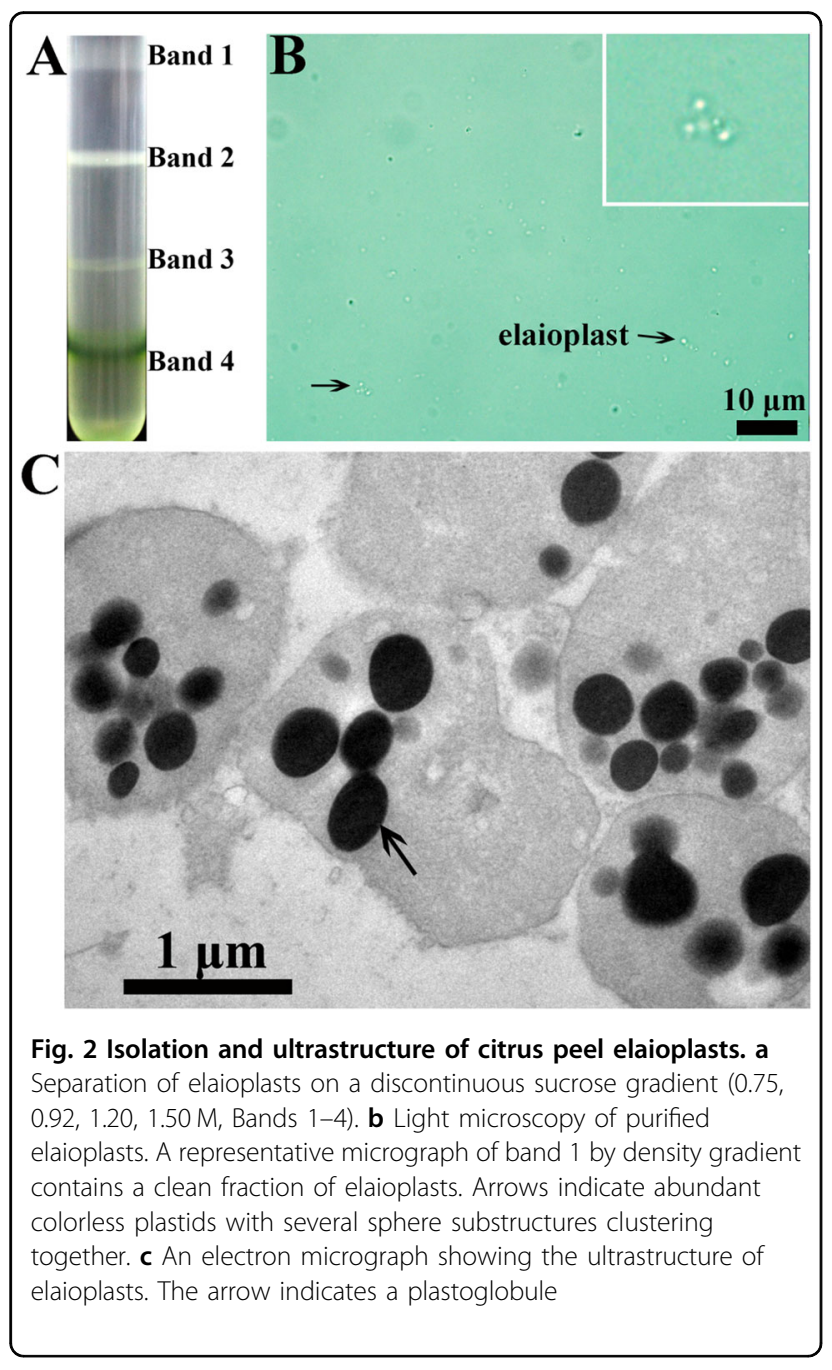

The purity of the elaioplast-enriched protein fractions was monitored by immunoblot analysis with antibodies against marker proteins for the plastid stroma (RbcL), cytosol (UDPase), vacuole (V-ATPase), and mitochondrion (VDAC1), as well as chloroplast (Lhca1 and Lhca4). As shown in Fig. 3, proteins extracted from the purified elaioplast fraction readily reacted with the antibody of RbcL. By contrast, no reaction could be detected with the cytosolic UGPase, mitochondrial VDAC1, or vacuolar VATPase. Additionally, no reaction was detected with antibodies to PSI type I chlorophyll a/b-binding proteins (Lhca1 and Lhca4) involved in photosynthesis within the chloroplasts. Reactions with these antibodies were observed in the total proteins extracted from the kumquat peel which contained both chloroplasts and elaioplasts. The above results suggest that the isolated elaioplasts used for the subsequent proteome analysis were of high purity without contamination by mitochondria, cytosol, vacuole, and chloroplasts.

\section{An inventory of proteins in elaioplasts by functional classification and subcellular localization}

We conducted a comprehensive proteomic analysis to characterize the proteins found in elaioplasts. A total of 1239 proteins with at least two detected peptides in two bio-replicates, were identified from purified elaioplasts. The raw data obtained from the elaioplast proteins were refined by searches against five plastid databanks (Plprot, AT-CHLORO, SUBA, PPDB, and Uniprot) and by three targeting predictors (WoLF PSORT, Predotar, and Target $\mathrm{P})$. Based on the chosen criteria for plastid-localized proteins (Materials and Methods), an inventory of 655 putative plastid proteins was constructed, accounting for $52 \%$ of all identified proteins (Supplementary Table S1). Among them, 562 proteins (accounting for $85.8 \%$ of these identified proteins) were predicted to be plastid targeted by at least one predictor program. WoLF PSORT, Target $\mathrm{P}$, and Predotar predicted plastid-localization of $76.0 \%$ (498 proteins), 38.2\% (250 proteins), and 31.9\% (209 proteins) of the 655 identified proteins, respectively. Two hundred and twenty-nine proteins (35.0\%) were predicted to be plastid targeted by at least two predictors, suggesting that these proteins are highly likely to be plastid localized. As shown in Supplementary Table S1 and in Fig. 4, the 655 putative elaioplast proteins were categorized into 32 functional classes according to MapMan bins.

\section{Major metabolic functions in the citrus elaioplast proteome}

The identification of proteome profiles allows an overview of proteins involved in various metabolic pathways ${ }^{8,24-28}$. As shown in Fig. 4, the major metabolic pathways in citrus elaioplasts are protein metabolism and process, transport, secondary metabolism, lipid 

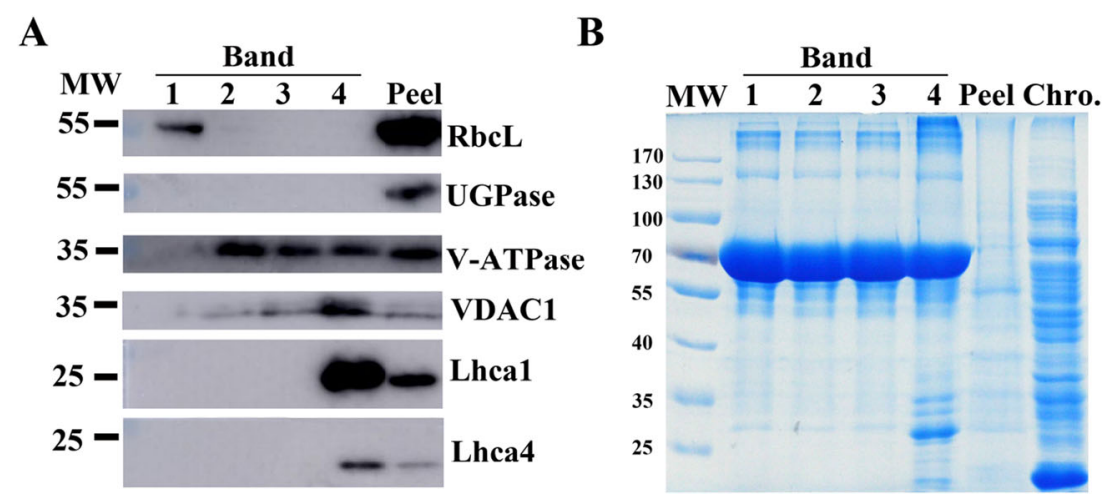

Fig. 3 Assessment of the purity of isolated elaioplasts using immunoblots. Different fractions of the sucrose gradient (Band 1 to Band 4) are compared to peel proteins using antibodies for plastid stroma large Rubisco subunit (RbcL), cytosolic UGPase, vacuolar (v)-ATPase, mitochondrial voltage-dependent anion-selective channel protein 1 (VDAC1), and photosynthesis Light-harvesting complex (Lhca1 and Lhca4); b Coomassie blue protein profiles of purified elaioplasts (Band 1 to Band 4) from kumquat peel, as compared with purified chromoplast (Chro.) from sweet orange flesh and total kumquat peel proteins

metabolism, redox and stress, amino acid metabolism, photosynthesis, DNA and RNA, and miscellaneous.

Elaioplasts are a type of heterotrophic plastid, wherein all the metabolic precursors are either generated via oxidative metabolism or actively imported from the cytosol to sustain anabolic reactions ${ }^{29}$. The proteins of the Calvin cycle are involved in providing intermediates for glycolysis, and proteins of the oxidative pentose phosphate pathway (OPPP) serve as a major source of reducing power for various metabolic pathways. As expected, we identified several proteins involved in the Calvin cycle within elaioplasts, such as fructose-bisphosphate aldolase, phosphoglycerate kinase, and transketolase. Furthermore, proteins participated in oxidative metabolism were well presented, including glucose-6-phosphate dehydrogenase, 6-phosphogluconolactonase, and 6-phosphogluconate dehydrogenase. Of note, transketolase and transaldolase (key enzymes of the non-oxidative part of OPPP), which are reported to feed the processes of anabolism or glycolysis $^{30}$, were also identified in the present study. Glycolysis also occurs in plastids to generate ATP and pyruvate in plants ${ }^{31}$. Several glycolysis enzymes including aldolase, phosphoglycerate kinase, enolase, and pyruvate kinase were observed. Consistent with previous findings in non-photosynthetic plastids, a substantial number of proteins responsible for the generation of ATP and

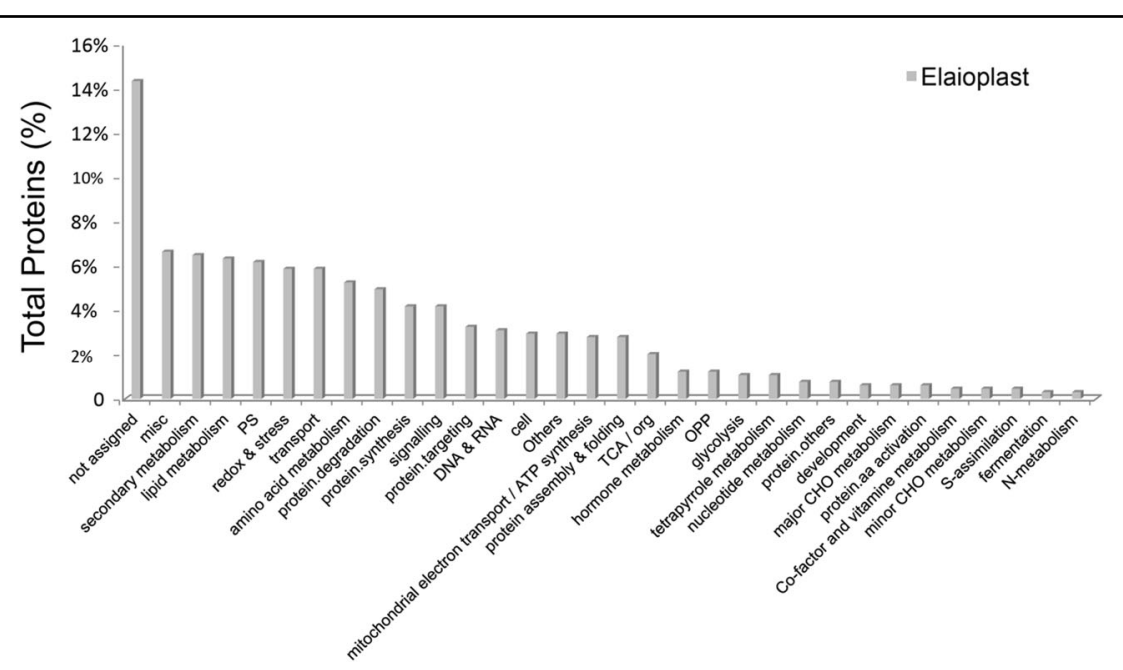

Fig. 4 Functional categories of proteins identified from elaioplasts. Proteins were categorized into 32 functional classifications according to MapMan (verified by PPDB, https://mapman.gabipd.org/mapman) 
reducing power were observed ${ }^{8}$, indicating their high conservation among different plastid types. Taken together, the observation of many proteins involved in essential biosynthetic activities suggests an active metabolism in elaioplasts to produce reducing power and energy, as well as the supply of precursors for the biosynthesis of metabolites.

Several proteins involved in photosynthesis were found in elaioplasts although the presence of chloroplasts was not observed using light microscopy and there was no signal obtained by immunoblotting using two photosynthesis-related antibodies (Fig. 3a). This suggests that these proteins serve roles in processes other than photosynthesis in elaioplasts. It is no surprise to find photosynthetic proteins in elaioplasts, since similar observations have been made in other types of nonphotosynthetic plastids, such as chromoplasts ${ }^{6,10}$ and amyloplasts $^{26}$.

FA are known to be synthesized in plastids ${ }^{28}$. We detected several enzymes for the synthesis of FA as well as glycolipids and phospholipids in elaioplasts, such as acetyl E1 beta pyruvate dehydrogenase complex, E2dihydrolipoamide acetyltransferase, E3-dihydrolipoamide dehydrogenase 1, long-chain acyl-CoA synthetase 7/8/9, and plastidial ketoacyl-ACP reductase (Supplementary Table S1). The accumulated FA could be converted into phosphatidic acid and/or imported into plastids via the trigalactosyldiacylglycerol (TDG) complex transporter, which is targeted to the plastid envelope membrane ${ }^{32}$. As expected, several key enzymes involved in lipid metabolism and homeostasis were detected in elaioplasts, such as TDG2, phosphatidylglycerolphosphate synthase 1, sulfoquinovosyldiacylglycerol synthase, as well as 3-ketoacylCoA thiolase, enoyl-CoA hydratase, phospholipase D $\alpha 1$, and cyclopropane-fatty-acyl-phospholipid synthase. These results indicate that the elaioplasts have the ability to synthesize FAs and polar lipids that might relate to the formation of new membranes during elaioplast development $^{33}$. This finding is also in agreement with a recent metabolic analysis in isolated tomato fruit chromoplasts using radiolabeled precursors ${ }^{34}$, which suggests a highly conservative mechanism for lipid biosynthesis and homeostasis in both chromoplasts and elaioplasts. It has been well documented that lipoxygenases (LOX), which are associated with generation of polyunsaturated FA hydroperoxides, could contribute to the biosynthesis of jasmonic acid (JA) ${ }^{35}$ and FA-derived flavor compounds ${ }^{36}$. In the present study, several enzymes involved in the LOX pathway (such as LOX1, LOX2, and LOX6) and JA metabolism (allene oxide cyclase 4) were detected. Therefore, it is possible that the presence of lipoxygenases in citrus elaioplasts and chromoplasts ${ }^{10}$ may play a key role in generating odor or flavor through production of LOX-derived volatiles in the outer peel and flesh of a fruit, respectively. A similar role for lipoxygenases in chromoplasts of tomato has been proposed ${ }^{6}$.

Elaioplasts are also important sites for amino acids synthesis. A large number of proteins responsible for amino acid metabolism were identified in elaioplasts, such as threonine synthase, aspartate aminotransferase, alanine transaminase, argininosuccinate synthase, cystathionine beta-lyase, 3-dehydroquinate synthase, isopropylmalate isomerase, isopropylmalate dehydrogenase, and ketol-acid reductoisomerase (Supplementary Table S1). Consistently, proteins of amino acid metabolism were also extensively found in non-photosynthetic plastids ${ }^{8,10,26}$. Taken together, our data suggest that these proteins involved in the biosynthesis of several types of amino acids, including aromatic amino acids have important functions in the active synthesis of amino acids within the elaioplasts.

Active redox system is a key regulator for plant development and fruit senescence and has a wide range of functions in gene expression, enzyme activities, and protein import in plastids ${ }^{37}$. In the elaioplast proteome, proteins involved in redox \& stress constituted one of the top five functional classes. Several essential components in maintaining homeostasis of cell redox were found in the elaioplast proteome. These include the proteins involved in ascorbate-glutathione (AsA-GSH) cycle and antioxidant system of plastids, such as glutathione peroxidases, peroxiredoxins (PRXs), superoxide dismutases (SODs), and thioredoxin. Additionally, several key enzymes involved in second active oxygen species scavenging system were identified in elaioplasts, including the AsA-GSH cycle catalyzed by ascorbate peroxidases (APXs), as well as the AsA-dependent generation systems catalyzed by (momo)dehydroascorbate reductase ${ }^{38}$. Like in photosynthetic chloroplasts, APXs encountered in elaioplasts may indicate the presence of the AsAdependent system involves the reduction of $\mathrm{H}_{2} \mathrm{O}_{2}$ using AsA as reductant ${ }^{39}$. The activation of plastid SOD expression was detected to be dependent on a copper chaperone for $\mathrm{SOD}^{40}$. Thus, as expected, Fe-SOD and $\mathrm{Mn}$-SOD were detected and their overexpression induced an enhancement of plant tolerance in response to environmental stresses $^{41,42}$. The presence of a large number of enzymes involved in oxidative stress response indicates a functionally active redox system in citrus elaioplasts and suggests the roles of these proteins in ROS metabolism, allowing the plastid to adapt to various environmental signals and processes during elaioplast development as fruit maturation proceeds.

Protein synthesis, degradation, and translocation regulate the biogenesis of plastids. In this study, proteins associated with import, ribosome assembly and proteases account for $16.9 \%$ of the total plastid proteins (Fig. 4). (1) Import of nuclear-encoded precursor proteins into 
Table 1 Thirty most abundant proteins identified from elaioplasts

\begin{tabular}{|c|c|c|c|c|c|c|c|}
\hline Accession & Rep. 1 & Rep. 2 & Protein description & Mapman & $\begin{array}{l}\text { iBAQ in } \\
\text { Rep. } 1\end{array}$ & $\begin{array}{l}\text { iBAQ in } \\
\text { Rep. } 2\end{array}$ & $\begin{array}{l}\text { Average } \\
\text { iBAQ }\end{array}$ \\
\hline Cs7g01840 & 13 & 11 & ADP, ATP carrier protein 2 & Transport & 27.7 & 27.4 & 27.6 \\
\hline Cs1g10270 & 27 & 28 & $\begin{array}{l}\text { Obsolete gene model variant (ATPase } 70 \\
\mathrm{kDa} \text { ) }\end{array}$ & Transport & 27.5 & 27.4 & 27.4 \\
\hline Cs3g24640 & 5 & 7 & OEP24-II & Transport & 27.0 & 26.8 & 26.9 \\
\hline orange1.1t02891 & 4 & 4 & HP30-2 (PRAT family) & Transport & 26.7 & 26.7 & 26.7 \\
\hline Cs2g14350 & 12 & 12 & Phosphate transporter (PHT3-1 or PIC1) & Transport & 26.6 & 26.7 & 26.7 \\
\hline Cs6g15300 & 42 & 44 & ATP-binding cassette transporter & Transport & 26.7 & 26.6 & 26.6 \\
\hline Cs2g13550 & 24 & 25 & $\mathrm{H}^{+}$-transporting ATP synthase beta chain & $\begin{array}{l}\text { Electron transport/ATP } \\
\text { synthesis }\end{array}$ & 28.6 & 28.7 & 28.6 \\
\hline orange1.1t01329 & 2 & 2 & ATP synthase epsilon chain & $\begin{array}{l}\text { Electron transport/ATP } \\
\text { synthesis }\end{array}$ & 27.5 & 27.4 & 27.4 \\
\hline Cs1g20530 & 15 & 15 & $\begin{array}{l}\text { 1-deoxy-D-xylulose 5-phosphate synthase } \\
\text { (DXS1) }\end{array}$ & Secondary metabolism & 27.7 & 27.9 & 27.8 \\
\hline orange1.1t03905 & 1 & 1 & (R)-limonene synthase 1 & Secondary metabolism & 27.9 & 27.5 & 27.7 \\
\hline Cs6g17510 & 11 & 11 & $\begin{array}{l}\text { Geranylgeranyl pyrophosphate synthase } \\
\text { (GGPS1) }\end{array}$ & Secondary metabolism & 27.5 & 27.4 & 27.5 \\
\hline Cs8g16700 & 29 & 28 & $\begin{array}{l}\text { 4-hydroxy-3-methylbutyl diphosphate } \\
\text { synthase (HDS) }\end{array}$ & Secondary metabolism & 26.8 & 26.8 & 26.8 \\
\hline Cs5g33860 & 9 & 10 & Thioredoxin family protein & Redox and stress & 27.0 & 27.2 & 27.1 \\
\hline Cs7g16410 & 7 & 7 & NADH-cytochrome b5 reductase & Redox and stress & 26.6 & 26.5 & 26.5 \\
\hline Cs6g15550 & 8 & 6 & Peroxiredoxin IIE (Prx\| E) & Redox and stress & 26.6 & 26.4 & 26.5 \\
\hline gi|113952630 & 17 & 19 & $\begin{array}{l}\text { Ribulose-1,5-bisphosphate carboxylase/ } \\
\text { oxygenase large subunit }\end{array}$ & PS & 29.3 & 29.6 & 29.4 \\
\hline Cs2g03780 & 6 & 6 & LHCII-1.2 & PS & 29.3 & 29.5 & 29.4 \\
\hline Cs6g18680 & 9 & 9 & Rubisco small subunit 3b & PS & 28.4 & 28.4 & 28.4 \\
\hline orange 1.1 t00226 & 15 & 14 & Transketolase-2 (TKL-2) & PS & 26.8 & 26.6 & 26.7 \\
\hline Cs6g14330 & 6 & 8 & 40 S ribosomal protein S14 (RPS14C) & Protein synthesis & 27.4 & 27.3 & 27.4 \\
\hline Cs5g16780 & 8 & 10 & Kunitz-type protease inhibitor & Protein degradation & 27.0 & 27.1 & 27.1 \\
\hline orange1.1t01459 & 31 & 31 & Cpn60 subunits & $\begin{array}{l}\text { Protein assembly \& } \\
\text { folding }\end{array}$ & 27.4 & 27.5 & 27.4 \\
\hline Cs7g29010 & 20 & 24 & HSP70-1 & $\begin{array}{l}\text { Protein assembly \& } \\
\text { folding }\end{array}$ & 26.7 & 26.8 & 26.7 \\
\hline Cs2g28210 & 13 & 15 & Formate dehydrogenase & Others & 27.7 & 27.6 & 27.7 \\
\hline Cs3g27530 & 3 & 2 & Hp17 & Not assigned & 27.1 & 27.0 & 27.1 \\
\hline Cs2g21000 & 17 & 14 & Hydroperoxide lyase1 & Not assigned & 26.9 & 27.0 & 27.0 \\
\hline Cs2g02520 & 13 & 14 & Fibrillin 1b (FBN1b) & Misc & 27.1 & 27.1 & 27.1 \\
\hline orange1.1t04376 & 31 & 35 & Lipoxygenase AtLOX2, plastid & Hormone metabolism & 27.9 & 27.7 & 27.8 \\
\hline Cs8g03410 & 9 & 11 & $\begin{array}{l}\text { Branched-chain amino acid transaminase } \\
2\end{array}$ & Amino acid metabolism & 26.4 & 26.5 & 26.4 \\
\hline Cs9g14610 & 9 & 8 & BCL-2-associated athanogene 7 (ATBAG7) & Signaling & 27.4 & 27.3 & 27.4 \\
\hline
\end{tabular}


plastids is mediated by translocon complexes at the outer and inner envelope membrane of chloroplasts (TOC/TIC complexes $)^{43}$, which is important for the biogenesis of either chloroplasts ${ }^{44}$ or chromoplasts ${ }^{1}$. However, the import machinery remains unclear in elaioplasts. In this study, numerous translocons including TOC33/75/159 and TIC62/55/40/22/110 in the protein import machinery were identified in elaioplast proteome (Supplementary Table S1). Among the TOC complex members, TOC75, which is deeply embedded within outer membrane of plastid, plays a major role to form protein translocation channel $^{43}$. TIC110, the major translocon component for protein translocation, plays a critical role in protein import into plastids ${ }^{45}$. The extensive identification of TOC/TIC complex likely plays an important role in elaioplast biogenesis on basis of protein import machinery. (2) Thirty ribosomal proteins of translation machinery were identified in citrus elaioplasts. These proteins are represented by plastid-encoded $30 \mathrm{~S}$ and $50 \mathrm{~S}$ subunit, together with nucleus-encoded $40 \mathrm{~S}$ and $60 \mathrm{~S}$ subunit. These findings suggest the presence of translation machinery in elaioplast biogenesis. (3) Maintaining a balance between protein synthesis, import, turnover, and processing is an important factor determining plastid fate and maintaining their homeostasis ${ }^{1,46}$. As expected, we identified numerous caseinolytic peptidase (Clp) proteases in the study (Supplementary Table S1), which have been well reported to form a chaperone complex to drive protein import, processing, and recycle plastid components. The knock-out of most members of Arabidopsis Clp proteases resulted in variegated/pale-green leaf phenotype, indicating their crucial roles in chloroplast development ${ }^{47}$. Elaioplast proteome also consists of several membrane-bound ATP-dependent metalloproteases (FtsH2, FtsH4, FtsH5, FtsH9, and FtsHi4), which have been described as critical for plastid development ${ }^{48}$. The extensive presence of $\mathrm{Clp}$ protease family in elaioplasts might contribute to elaioplast biogenesis by processing and/or cleavage of transit peptides of proteins in the plastids.

\section{Abundant proteins in citrus elaioplasts}

The protein abundance for all proteins detected in kumquat elaioplasts was determined based on intensitybased absolute quantification (iBAQ). The 30 most abundant proteins of kumquat elaioplasts are listed in Table 1. Among them, we identified four enzymes involving in secondary metabolism. Three enzymes (i.e. DXS, HDS, and GGPS) belong to the 2-C-methyl-D-erythritol 4phosphate (MEP) pathway, which provides precursors to synthesize monoterpenes within plastids. The fourth (orange1.1t03905) was a putative terpene synthase, which might be responsible for the accumulation of D-limonene (accounting for up to $90 \%$ among monoterpene compounds) in outer peel of citrus ${ }^{49,50}$. The importance of DXS in regulating MEP substrate flux in kiwifruit and grape has previously been reported ${ }^{51,52}$ and the identification of MEP pathway proteins and TPS genes in citrus elaioplasts indicates the key role that elaioplasts play in basic metabolism.

Eight proteins involved in transport or electron transport categories, such as ADP, ATP carrier protein, ATPbinding cassette transporter, ATPase, as well as ATP synthase beta/epsilon chain were also detected in kumquat elaioplasts. ATPase acts as an essential regulator of membrane trafficking, which couples free energy generated from ATP hydrolysis to transport of substances across the membranes of many organelles ${ }^{53}$. ATP synthases are involved in energy production and proton transport. The identification of these proteins at high abundance suggests they may play an important role in active energy production and transport during elaioplast development. The presence of an active ATP generation system that is also necessary for the formation of membranes in plastids ${ }^{54}$. Elevated energy production has also be observed in other heterotrophic plastids, such as chromoplasts $^{6}$ and proplastids ${ }^{55}$.

\section{The citrus elaioplast proteome compared with citrus chromoplast proteome}

To determine whether the ultrastructure was different between elaioplasts and chromoplasts, we purified chromoplasts from citrus flesh for comparison with elaioplasts. TEM analysis showed that the size of elaioplasts was $2.15 \pm 0.70 \mu \mathrm{m}$ in diameter, which was significantly smaller than that of chromoplast $(2.59 \pm 0.60 \mu \mathrm{m})$ (Fig. 5a; Supplementary Figure S2). The number of plastoglobules $(5.86 \pm 2.65)$ per elaioplast was approximately half that per chromoplast $(11.59 \pm 6.94)$ (Fig. 5b). However, the size of plastoglobules within elaioplasts was $0.48 \pm 0.25$ $\mu \mathrm{m}$ in diameter, which was about threefold greater than the diameter of plastoglobules within a chromoplast $(0.14$ $\pm 0.06 \mu \mathrm{m}$ ) (Fig. 5c). Taken together, the above results suggest that there are differences in ultrastructure between elaioplasts and chromoplasts.

Given the structural differences between elaioplasts and chromoplasts, it was not surprising that differences in protein distribution patterns on SDS-PAGE gels were also observed (Fig. 3b). We further explored these differences by comparing the elaioplast proteome with that of chromoplast at the mass spectrometric level (Supplementary Figure S3). Distinct proteome profiles for the biosynthesis and accumulation of different secondary metabolites was observed between elaioplasts and chromoplasts. A total of 42 and 40 core enzymes involved in secondary metabolism were identified in elaioplasts and chromoplasts, respectively (Supplementary Table S2). The MEP pathway provides substrates to synthesize terpenes, tocopherols, 

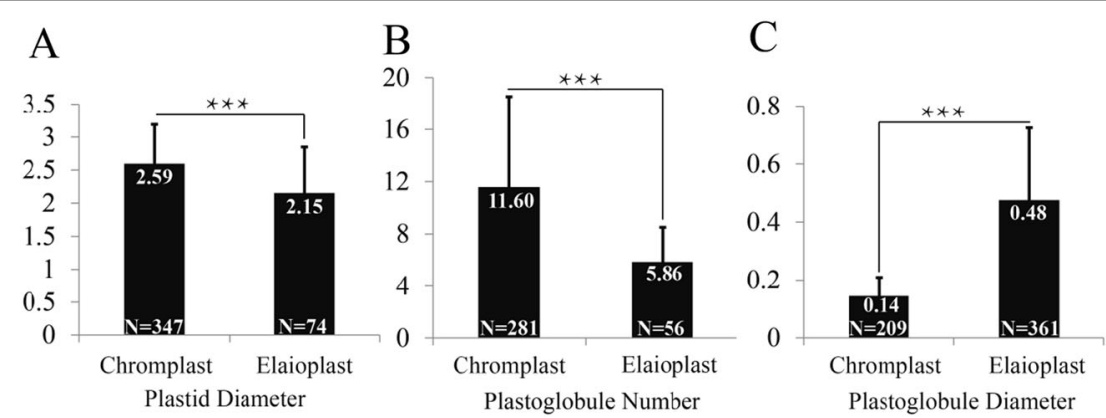

Fig. 5 Ultrastructural differences between elaioplasts and chromoplasts. a The size of elaioplasts and chromoplasts in diameter. $\mathbf{b}$ The number of plastoglobules versus that of chromoplasts. $\mathbf{c}$ The size of plastoglobule of elaioplasts versus that of chromoplasts. Data are indicated as means \pm SD and $p$ values were calculated using Student's $t$ test in Microsoft Excel. ${ }^{* *} p<0.001$; number $(N)$ in the bars represents the number of plastids measured by using ImageJ software

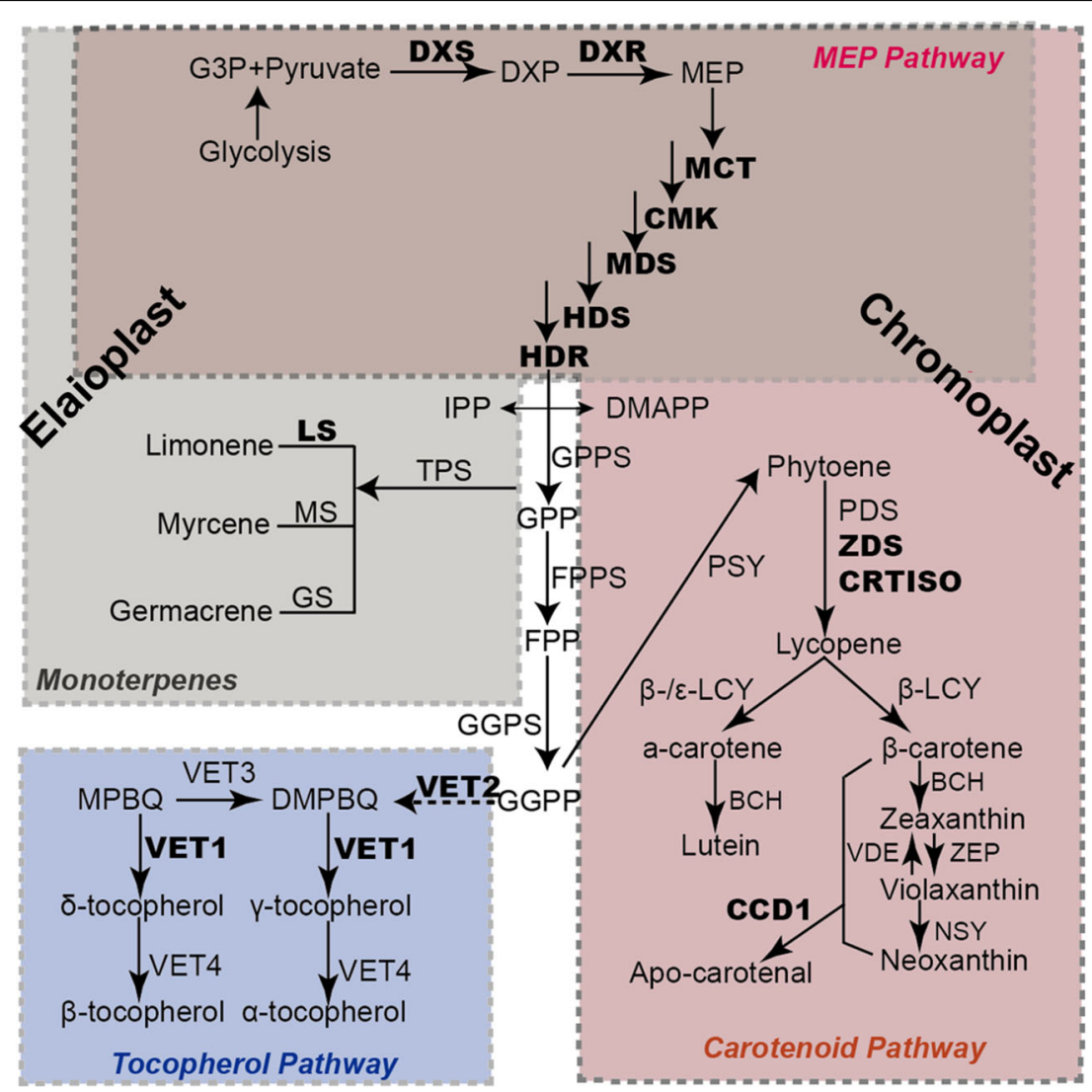

Fig. 6 Schematic illustration of the biosynthesis of secondary metabolites in elaioplasts and chromoplasts. Proteins in boldface were identified in the proteomes of both plastid types. The full name of enzymes was listed in Supplementary Table S2. DXP 1-deoxy-D-Xylulose 5phosphate, MEP 2C-methylerythritol 4-phosphate, IPP isopentenyl diphosphate, DMAPP dimethylallyl pyrophosphate, GPP geranyl diphosphate, FPP farnesyl disphosphate, GGPP geranylgeranyl diphosphate, MPBQ 2-Methyl-6-phytyl-1,4-hydroquinol, DMPBQ 2,3-Dimethyl-5-phytyl-1,4-hydroquinol

and carotenoids (Fig. 6). In this study, an entire set of enzymes responsible for the MEP pathway were found in both elaioplasts and chromoplasts, including DXS, DXR, CMK, MCT, MDS, HDS, and HDR. Terpenoids are specifically accumulated in elaioplasts. We found seven proteins involved in terpenoid biosynthesis, such as myrcene synthase, 1,8-cineole synthase, germacrene synthase, and limonene synthase which accounted for more 
than $16.7 \%$ of total proteins involved in secondary metabolism (Supplementary Table S3). By contrast, only limonene synthase (accounting for 2.5\%) was identified in chromoplasts. With regard to carotenoid metabolism, ten enzymes (accounting for 25\%) involved in carotenoid synthesis were identified in the chromopasts, including the rate limiting enzyme phytoene synthase, $\zeta$-carotene desaturase (ZDS), phytoene desaturase, lycopene $\beta$ cyclase, carotenoid isomerase (CRTISO), and $\beta$-carotene hydroxylase. By contrast, only CRTISO and ZDS were identified in elaioplasts. The synthesis of volatile apocarotenoids enhances dramatically during plastid development $^{56,57}$. Consistently, CCD1 was detected in elaioplasts and chromoplasts.

The extensive formation of plastoglobules within chromoplasts of citrus flesh is the most representative feature during chromoplast biogenesis ${ }^{9}$, which is confirmed by the present study (Fig. 5b). Thirty plastoglobule-localized proteins have been established in Arabidopsis ${ }^{58}$, and in our previous chromoplast proteome analysis we identified citrus homologs for 24 of these 30 proteins ${ }^{9}$. In this study, we identified five additional plastoglobule-localized proteins (Supplementary Table S4). However, it remains unclear whether plastoglobules within elaioplasts have a protein composition similar to that in chromoplasts. In elaioplasts, we only identified eight proteins that are predicted to be plastoglobule-localized using the same method. They include fibrillin 1 (FBN1), FBN7, tocopherol cyclase, flavin reductase-related 1/2, PLAT/LH2-1, and diacylglycerol acyltransferase. The FBN family is required for the development of leaf and fruit, and is involved in regulation of the extension of plastoglobules ${ }^{58}$. In pepper, it is reported to be important for the formation of carotenoidlipoprotein sequestration substructures and contributes to elevated levels of carotenoid accumulation ${ }^{59}$. Fibrillin and its homologs were identified at high abundance in elaioplasts (Table 1), suggesting a possible role in the formation of plastoglobules. Since lipid-rich colorless elaioplasts likely have specialized but unknown functions $^{60}$, they might possess a unique plastoglobule proteome. Therefore, the further determination of plastoglobule proteomes of this colorless, nonphotosynthetic plastid is necessary and meaningful.

\section{Conclusion}

The present study reveals several important characteristics of the elaioplasts of kumquat peel. An inventory of 655 candidate proteins predicted to be localized in citrus elaioplasts was constructed based on in silico homology analyses, and the majority of these proteins share similar distribution patterns in Mapman functional classes between elaioplast and chromoplast. The identification of the abundant proteins associated with the MEP pathway and terpene biosynthesis in elaioplasts suggest that this biochemical process may define a basic feature of elaioplasts. In conclusion, this proteomic analysis provides a comprehensive understanding of citrus elaioplasts as a key step towards uncovering the biogenesis of the elaioplast and its terpene metabolism.

\section{Acknowledgments}

This work was supported by the National Natural Science Foundation of China (NSFC, grant nos. 31501739), the National Basic Research Program of China (973 project No. 2013CB127105), and Huazhong Agricultural University Scientific \& Technological Self-innovation Foundation (No. 2662015BQ034). We are grateful to Prof. Zuoxiong Liu (Foreign Language College of Huazhong Agricultural University), Ross Atkinson (Plant and Food research, New Zealand), and Prof. Shunyuan Xiao (Center for Biosystems Research, University of Maryland Biotechnology Institute, USA) for their critical review of the manuscript.

\section{Author details}

${ }^{1}$ Key Laboratory of Horticultural Plant Biology (Ministry of Education), College of Horticulture and Forestry Science, Huazhong Agricultural University, Wuhan 430070, China. ${ }^{2}$ Institute of Citrus Science, Huazhong Agricultural University, Wuhan 430070, China. ${ }^{3}$ Shanghai Applied Protein Technology Co. Ltd, Shanghai 200233, China. ${ }^{4}$ Guangxi Citrus Research Institute, Guangxi 541004, China

\section{Conflict of interest}

The authors declare that they have no conflict of interest.

\section{Publisher's note}

Springer Nature remains neutral with regard to jurisdictional claims in published maps and institutional affiliations.

Supplementary Information accompanies this paper at (https://doi.org/ 10.1038/s41438-017-0014-X).

Received: 13 August 2017 Revised: 7 December 2017 Accepted: 10 December 2017

Published online: 07 February 2018

\section{References}

1. Egea, I. et al. Chromoplast differentiation: current status and perspectives. Plant Cell. Physiol. 51, 1601-1611 (2010).

2. van Wijk, K. \& Baginsky, S. Plastid proteomics in higher plants: current state and future goals. Plant Physiol. 155, 1578-1588 (2011).

3. Li, L., Yuan, H., Zeng, Y. \& Xu, Q. Plastids and carotenoid accumulation. Subcell. Biochem. 79, 273-93 (2016).

4. Millar, A. H., Whelan, J. \& Small, I. Recent surprises in protein targeting to mitochondria and plastids. Curr. Opin. Plant Biol. 9, 610-615 (2006).

5. Siddique, M. A., Grossmann, J., Gruissem, W. \& Baginsky, S. Proteome analysis of bell pepper (Capsicum annuum L.) chromoplasts. Plant Cell. Physiol. 47, 1663-1673 (2006).

6. Barsan, $C$. et al. Characteristics of the tomato chromoplast revealed by proteomic analysis. J. Exp. Bot. 61, 2413-2431 (2010).

7. Barsan, C. et al. Proteomic analysis of chloroplast-to-chromoplast transition in tomato reveals metabolic shifts coupled with disrupted thylakoid biogenesis machinery and elevated energy-production components. Plant Physiol. 160, 708-725 (2012).

8. Wang, Y.-Q. et al. Proteomic analysis of chromoplasts from six crop species reveals insights into chromoplast function and development. J. Exp. Bot. 64 949-961 (2013).

9. Zeng, Y. et al. A comprehensive analysis of chromoplast differentiation reveals complex protein changes associated with plastoglobule biogenesis and remodeling of protein systems in sweet orange flesh. Plant Physiol. 168, 1648-1665 (2015). 
10. Zeng, Y. et al. A proteomic analysis of the chromoplasts isolated from sweet orange fruits Citrus sinensis (L.) Osbeck. J. Exp. Bot. 62, 5297-5309 (2011).

11. Zeng, Y. et al. Phosphoproteomic analysis of chromoplasts from sweet orange during fruit ripening. Physiol. Plant. 150, 252-270 (2014).

12. Eastmond, P. J., Dennis, D. T. \& Rawsthorne, S. Evidence that a malate/inorganic phosphate exchange translocator imports carbon across the leucoplast envelope for fatty acid synthesis in developing castor seed endosperm. Plant Physiol. 114, 851-856 (1997).

13. Yamasaki, Y. \& Akimitsu, K. In situ localization of gene transcriptions for monoterpene synthesis in irregular parenchymic cells surrounding the secretory cavities in rough lemon (Citrus jambhiri). J. Plant Physiol. 164, 1436-1448 (2007).

14. Michel, G., Ginette, P., Jean-Pierre, C., Anne, M. \& Colette, B.-D. Monoterpene hydrocarbon biosynthesis by isolated leucoplasts of Citrofortunella mitis. Planta 159, 373-381 (1983).

15. Nagegowda, D., Gutensohn, M., Wilkerson, C. \& Dudareva, N. Two nearly identical terpene synthases catalyze the formation of nerolidol and linalool in snapdragon flowers. Plant J. 55, 224-239 (2008).

16. Gupta, R. \& Singh, R. Fatty acid synthesis in leucoplasts isolated from developing seeds of Brassica campestris. J. Plant Biochem. Biot. 5, 127-130 (1996).

17. Yadav, S. \& Singh, R. Fatty acid synthesis in isolated leucoplasts of developing seeds of Brassica campestris L. Acta Physiol. Plant 25, 235-240 (2003).

18. Lara-Núñez, A. Characterization of a dicarboxylate exchange system able to exchange pyrophosphate for L-malate in non-photosynthetic plastids from developing maize embryos. Plant Sci. 166, 1335-1343 (2004).

19. Voo, S., Grimes, H. \& Lange, B. Assessing the biosynthetic capabilities of secretory glands in Citrus peel. Plant Physiol. 159, 81-94 (2012).

20. Tao, N., Jia, L. \& Zhou, H. Anti-fungal activity of Citrus reticulata Blanco essential oil against Penicillium italicum and Penicillium digitatum. Food Chem. $\mathbf{1 5 3}$ 265-271 (2014).

21. Patrignani, F., Siroli, L., Serrazanetti, D., Gardini, F. \& Lanciotti, R. Innovative strategies based on the use of essential oils and their components to improve safety, shelf-life and quality of minimally processed fruits and vegetables. Trend Food Sci. Tech. 46, 311-319 (2015).

22. Eilati, S., SP, M. \& Budowski, P. Seasonal development of external color and carotenoid content in the peel of ripening 'Shamouti' oranges. J. Am. Soc. Hortic. Sci. 94, 346-348 (1969).

23. Iglesias, D. J. et al. Physiology of citrus fruiting. Braz. J. Plant Physiol. 19, 333-362 (2007).

24. Kleffmann, T. et al. The Arabidopsis thaliana chloroplast proteome reveals pathway abundance and novel protein functions. Curr. Biol. 14, 354-362 (2004).

25. von Zychlinski, A. et al. Proteome analysis of the rice etioplast metabolic and regulatory networks and novel protein functions. Mol. Cell. Proteom. 4, 1072-1084 (2005).

26. Balmer, Y., Vensel, W., DuPont, F., Buchanan, B. \& Hurkman, W. Proteome of amyloplasts isolated from developing wheat endosperm presents evidence of broad metabolic capability. J. Exp. Bot. 57, 1591-1602 (2006).

27. Daher, Z. et al. Proteomic analysis of Medicago truncatula root plastids. Proteomics 10, 2123-2137 (2010).

28. Li, L. \& Yuan, H. Chromoplast biogenesis and carotenoid accumulation. Arch. Biochem. Biophys. 539, 102-109 (2013).

29. Neuhaus, H. \& Emes, M. Nonphotosynthetic metabolism in plastids. Annu. Rev. Plant Biol. 51, 111-140 (2000).

30. Stincone, A. et al. The return of metabolism: biochemistry and physiology of the pentose phosphate pathway. Biol. Rev. 90, 927-963 (2015).

31. Plaxton, W. The organization and regulation of plant glycolysis. Annu. Rev. Plant Biol. 47, 185-214 (1996).

32. Benning, C. Mechanisms of lipid transport involved in organelle biogenesis in plant cells. Annu. Rev. Cell. Dev. Biol. 25, 71-91 (2009).

33. Benning, C. A role for lipid trafficking in chloroplast biogenesis. Prog. Lipid Res. 47, 381-389 (2008).

34. Angaman, D. et al. Precursor uptake assays and metabolic analyses in isolated tomato fruit chromoplasts. Plant Methods 8, 1 (2012).

35. Wasternack, C. Jasmonates: An update on biosynthesis, signal transduction and action in plant stress response, growth and development. Ann. Bot. $\mathbf{1 0 0}$ 681-697 (2007).

36. Chen, $G$. et al. Identification of a specific isoform of tomato lipoxygenase (TomloxC) involved in the generation of fatty acid-derived flavor compounds. Plant Physiol. 136, 2641-2651 (2004).
37. Balsera, M., Soll, J. \& Buchanan, B. Redox extends its regulatory reach to chloroplast protein import. Trends Plant Sci. 15, 515-521 (2010).

38. Yabuta, Y. et al. Thylakoid membrane-bound ascorbate peroxidase is a limiting factor of antioxidative systems under photo-oxidative stress. Plant J. 32, 915-925 (2002)

39. Asada, K. Production and scavenging of reactive oxygen species in chloroplasts and their functions. Plant Physiol. 141, 391-396 (2006).

40. Huang, C., Kuo, W., Weiss, C. \& Jinn, T. Copper chaperone-dependent and -independent activation of three Copper-Zinc superoxide dismutase homologs localized in different cellular compartments in Arabidopsis. Plant Physiol. 158, 737-746 (2012)

41. Liu, X. et al. Over-expression of ScMnSOD, a SOD gene derived from Jojoba, improve drought tolerance in Arabidopsis. J. Integr. Agr. 12, 1722-1730 (2013).

42. McKersie, B., Murnaghan, J., Jones, K. \& Bowley, S. Iron-superoxide dismutase expression in transgenic alfalfa increases winter survival without a detectable increase in photosynthetic oxidative stress tolerance. Plant Physiol. 122, 1427-37 (2000)

43. Jarvis, P. Targeting of nucleus-encoded proteins to chloroplasts in plants. New Phytol. 179, 257-285 (2008).

44. Ling, Q. \& Jarvis, P. Functions of plastid protein import and the ubiquitinproteasome system in plastid development. Biochim. Et. Biophys. Acta 1847, 939-948 (2015)

45. Inaba, T. et al. Arabidopsis Tic110 is essential for the assembly and function of the protein import machinery of plastids. Plant Cell. 17, 1482-1496 (2005).

46. Sakamoto, W. Protein degradation machineries in plastids. Annu. Rev. Plant Biol. 57, 599-621 (2006).

47. Olinares, P., Kim, J. \& van Wijk, K. The Clp protease system; a central component of the chloroplast protease network. Biochim. Et. Biophys. Acta 1807, 999-1011 (2011).

48. Kato, Y. \& Sakamoto, W. New insights into the types and function of proteases in plastids. Int. Rev. Cell. Mol. Biol. 280, 185-218 (2010).

49. Zhang, $\mathrm{H}$. et al. Comprehensive comparative analysis of volatile compounds in citrus fruits of different species. Food Chem. 230, 316-326 (2017).

50. Rodríguez, A. et al. Terpene down-regulation triggers defense responses in transgenic orange leading to resistance against fungal pathogens. Plant Physiol. 164, 321-339 (2014).

51. Nieuwenhuizen, N. J. et al., Natural variation in monoterpene synthesis in kiwifruit: transcriptional regulation of terpene synthases by NAC and EIN3-like transcription factors. Plant Physiol. 167, 1243-58 (2015).

52. Duchêne, E. et al. A grapevine (Vitis vinifera L.) deoxy-d-xylulose synthase gene colocates with a major quantitative trait loci for terpenol content. Theor. Appl. Genet. 118, 541-552 (2009).

53. Schumacher, K. \& Krebs, M. The V-ATPase: small cargo, large effects. Curr. Opin. Plant Biol. 13, 724-730 (2010).

54. Morstadt, L. et al. Chemiosmotic ATP synthesis in photosynthetically inactive chromoplasts from Narcissus pseudonarcissus $L$. linked to a redox pathway potentially also involved in carotene desaturation. Planta $\mathbf{2 1 5}$, 134-140 (2002).

55. Brautigam, A. \& Weber, A. P. Proteomic analysis of the proplastid envelope membrane provides novel insights into small molecule and protein transport across proplastid membranes. Mol. Plant 2, 1247-61 (2009).

56. Simkin, A., Schwartz, S., Auldridge, M., Taylor, M. \& Klee, H. The tomato carotenoid cleavage dioxygenase 1 genes contribute to the formation of the flavor volatiles beta-ionone, pseudoionone, and geranylacetone. Plant J. 40, 882-892 (2004).

57. Ibdah, M. et al. Functional characterization of CmCCD1, a carotenoid cleavage dioxygenase from melon. Phytochemistry 67, 1579-1589 (2006).

58. Lundquist, P. et al. The functional network of the Arabidopsis plastoglobule proteome based on quantitative proteomics and genome-wide coexpression analysis. Plant Physiol. 158, 1172-92 (2012).

59. Deruère, J. et al. Fibril assembly and carotenoid overaccumulation in chromoplasts: a model for supramolecular lipoprotein structures. Plant Cell. 6, 119-133 (1994).

60. van Wijk, K. \& Kessler, F. Plastoglobuli: plastid microcompartments with integrated functions in metabolism, plastid developmental transitions, and environmental adaptation. Annu. Rev. Plant Biol. 68, 11.1-11.37 (2017). 